\section{Schmerzen nach totalem Kniegelenkersatz: Welche Rolle spielt das Geschlecht?}

Perruccio AV et al. Sex-Modified Effects of

Depression, Low Back Pain, and Comorbidities on

Pain After Total Knee Arthroplasty for Osteo-

arthritis. Arthritis Care Res (Hoboken) 2020; 72 :

1074-1080. doi: 10.1002/acr.24002

Obwohl der totale Kniegelenkersatz eine wirksame Therapie der Gonarthrose darstellt, berichtet ein erheblicher Anteil der operierten Patienten über nur geringfügige Verbesserungen oder sogar anhaltende Schmerzen. Welche präoperativen Faktoren für ein ungünstige Operationsergebnis prädisponieren und welche Rolle das Geschlecht diesbezüglich spielt, untersuchte nun ein Team kanadischer Forscher.

An der prospektiven Studie nahmen $477 \mathrm{~Pa}-$ tienten teil, welche aufgrund einer Kniegelenkarthrose mittels totalem Gelenkersatz behandelt wurden. In allen Fällen erfolgte die Implantation einer zementierten Prothese. Patienten mit einem Revisionseingriff oder einer posttraumatischen oder inflammatorisch bedingten Arthroseproblematik schlossen die Forscher von der Studienteilnahme aus. Alle Probanden wurden vor sowie 3 Monate nach der Operation mithilfe des KOOS (Knee Injury and Osteoarthritis Outcome Score) zu ihrer Schmerzbelastung befragt. Ferner erfassten die Wissenschaftler eine Reihe weiterer Parameter wie das Alter, das Geschlecht, Größe und Gewicht, den Bodymassindex, die Begleiterkrankungen, die Anzahl weiterer symptomatischer Körperregionen sowie das Vorliegen depressiver Symptome. Zusätzlich objektivierten sie intra- und postoperative Komplikationen und befragten die Studienteilnehmer zu seit dem Eingriff aufgetretenen weiteren unerwünschten Ereignissen.

\section{Ergebnisse}

Die Studienteilnehmer waren im Schnitt 65 Jahre alt, 57\% waren weiblich. Die Frauen berichteten im Vergleich zu den Männern sowohl vor als auch nach der Operation über signifikant stärkere Schmerzbelastungen. Sie litten zudem an ausgedehnteren Gelenkbeteiligungen und ausgeprägteren depressiven Symptomen. Bei einer einfachen Adjustierung bezüglich verschiedener Kovariablen stellten die Forscher keinen signifikanten Einfluss des Geschlechts auf die postoperative Schmerzintensität fest. Ein differenziertes Berechnungsmodell kam jedoch zu dem Ergebnis, dass der Einfluss verschiedener Kovariablen bezüglich der Schmerzbelastung nach 3 Monaten von der Geschlechtszugehörigkeit beeinflusst wurde: Das Vorliegen von mindestens 3 Komorbiditäten (versus < 3) ging lediglich bei Frauen mit signifikant stärkeren postoperativen Schmerzen einher. Rückenschmerzen sowie depressive Symptome begünstigten dagegen bei Männern stärkere Schmerzbelastungen. Stärkere präoperative Schmerzen führten bei Frauen und Männern gleichermaßen zu stärkeren postoperativen Schmerzbelastungen.

\section{FAZIT}

Wie stark sich verschiedene patientenspezifische Faktoren auf die frühe postoperative Schmerzbelastung nach totalem Kniegelenkersatz auswirkt, hängt offenbar vom Geschlecht des Patienten ab, schlussfolgern die Autoren. Für die Vorhersage der Erfolgschancen des Eingriffs spielt daher die Geschlechtszugehörigkeit eine entscheidende Rolle.

Dr. med. Judith Lorenz, Künzell 